\title{
Efficacy of granulocyte colony stimulating factor in patients with severe alcoholic hepatitis with partial or null response to steroid (GRACIAH trial): study protocol for a randomized controlled trial
}

Yuri $\mathrm{Cho}^{1 \dagger}$, Youn Su Park ${ }^{2 \dagger}$, Hwi Young Kim ${ }^{3}$, Won $\mathrm{Kim}^{2 *}$ (D, Heon Ju Lee ${ }^{4^{*}}$ and Dong Joon $\mathrm{Kim}^{5^{*}}$

\begin{abstract}
Background: Alcoholic hepatitis (AH) has the most severe presentation among alcohol-related liver diseases. Corticosteroids are the most widely recommended treatment for severe AH. However, more innovative, refined treatment measures are required because of its high mortality despite corticosteroid treatment. This study aims to determine whether granulocyte colony stimulating factor (G-CSF) treatment increases short-term survival in patients with severe $\mathrm{AH}$ refractory to corticosteroid treatment.

Methods/design: Patients with severe AH whose Maddrey's discriminant function (MDF) score is $\geq 32$ and who will be treated with prednisolone (40 mg/day) for 1 week will be screened. Among them, 190 subjects with a partial response (PR) (Lille score 0.16-0.56), and 78 subjects with a null response (NR) (Lille score $\geq 0.56$ ) will be enrolled. Subjects with PR will be randomized to steroid plus placebo or steroid plus $12 \mathrm{G}$-CSF injections $(5 \mu \mathrm{g} / \mathrm{kg} /$ day for 5 days followed by every 3 days) at a ratio of 1:1. Subjects with a NR will be randomized to the placebo or G-CSF group (1:1). Study subjects in the PR group will be treated with prednisolone for 28 days followed by dose tapering for an additional 2 weeks. The primary endpoint is the 2-month survival rate in the NR group and the 6-month survival rate in the PR group. Child-Turcotte-Pugh, model for end-stage liver disease score, and the change in the proportion of peripheral circulating CD34-positive cells will be analyzed as risk factors for mortality. Preliminary safety data for the initial 10 study subjects enrolled in the PR study will be assessed to determine whether the PR study would be continued, according to the G-CSF-mobilized, peripheral-blood stem cell donor assessment protocol of the National Marrow Donor Program.

(Continued on next page)
\end{abstract}

\footnotetext{
*Correspondence: drwon1@snu.ac.kr; heonjulee@yu.ac.kr; hjlee@med.yu.ac.kr; djkim@hallym.ac.kr

†Yuri Cho and Youn Su Park contributed equally to this work.

${ }^{2}$ Division of Gastroenterology and Hepatology, Department of Internal Medicine, Seoul Metropolitan Government Seoul National University Boramae Medical Center, 20, Boramae-ro 5-gil, Dongjak-gu, Seoul 07061, Republic of Korea

${ }^{4}$ Department of Internal Medicine, Yeungnam University College of Medicine, Yeungnam University Medical Center, 170, Hyeonchung-ro, Nam-gu, Daegu 42415, Republic of Korea

${ }^{5}$ Department of Internal Medicine, Hallym University College of Medicine, Chuncheon Hallym University Medical Center, 153 Gyo-dong, Chuncheon-si, Gangwon-do 24253, Republic of Korea

Full list of author information is available at the end of the article
}

(c) The Author(s). 2018 Open Access This article is distributed under the terms of the Creative Commons Attribution 4.0 International License (http://creativecommons.org/licenses/by/4.0/), which permits unrestricted use, distribution, and reproduction in any medium, provided you give appropriate credit to the original author(s) and the source, provide a link to the Creative Commons license, and indicate if changes were made. The Creative Commons Public Domain Dedication waiver (http://creativecommons.org/publicdomain/zero/1.0/) applies to the data made available in this article, unless otherwise stated. 
(Continued from previous page)

Discussion: We hypothesized that G-CSF would prolong short-term survival of patients with severe AH refractory to corticosteroid treatment. This is a proof-of-concept trial designed to assess the efficacy of Lille-score-guided G-CSF treatment. This trial is also designed to identify a special subgroup in whom G-CSF rescue treatment would improve liver function and prolong survival.

Trial registration: ClinicalTrials.gov, NCT02442180. Prospectively registered on 13 May 2015.

Keywords: Alcoholic hepatitis, Prednisolone, G-CSF, Discriminant function,

\section{Background}

Over several decades, South Korea has ranked as one of the developed countries with the largest consumption of alcohol in the world [1,2]. Alcohol-related liver disease is a broad-spectrum disease entity, including hepatic steatosis, hepatitis, cirrhosis, and hepatocellular carcinoma, as one individual can have various progressive stages of liver damage. Among them, alcoholic hepatitis has the worst prognosis, with short-term mortality of $40 \%$ within 1 month of onset [3-5].

Severe alcoholic hepatitis (AH) is defined as Maddrey's Discriminant Function (MDF) score $\geq 32$ points or when the patient also had hepatic encephalopathy, which has a very poor prognosis and 28-day mortality of $30-50 \%$ if not treated $[6,7]$. Severe $\mathrm{AH}$ has a higher short-term mortality rate than any other liver disease, including viral hepatitis and nonalcoholic fatty liver disease. Nonetheless, survival has not notably improved in severe $\mathrm{AH}$, despite advances in medicine [8].

Corticosteroids (prednisolone $40 \mathrm{mg} /$ day, 28 days) are the most widely recommended treatment for severe $\mathrm{AH}$. Corticosteroid treatment is indicated for patients with severe $\mathrm{AH}$ who are expected to have a very poor prognosis, with a MDF score $\geq 32$ points and model for end-stage liver disease (MELD) score $>21$ points or hepatic encephalopathy $[9,10]$. However, corticosteroids cannot be used when the patient also has upper gastrointestinal bleeding, renal failure, pancreatitis, or an uncontrolled infection [11, 12]. A recent meta-analysis of individual patient data from five randomized controlled trials demonstrated that the 28-day survival rate was significantly higher in the steroid group than in the placebo group ( $80.0 \%$ vs. $65.7 \%, P<0.001)$. Thus, corticosteroids are a standard treatment in selected patients with $\mathrm{AH}$ and a MDF score $\geq 32$ [7]. However, early recognition of non-response to corticosteroids $(40 \%$ of the patients with severe $\mathrm{AH}$ who receive corticosteroid treatment) is essential to minimize unnecessary exposure to corticosteroids [13]. The short-term mortality of $20 \%$ despite corticosteroid treatment still desperately requires more advanced treatment measures [7].

To overcome the difficulties of exploring rescue therapies for steroid non-responders, several clinical trials have been conducted to increase the survival rate using granulocyte colony stimulating factor (G-CSF) in patients with severe AH $[14,15]$. Although the standard treatment with corticosteroids may reduce necroinflammation in patients with $\mathrm{AH}$, mortality remains strikingly high [16]. Therefore, if G-CSF treatment facilitates liver regeneration and enhances neutrophil function in patients with severe $\mathrm{AH}$ taking corticosteroids [17-19], it is expected to significantly improve the current standard treatment strategy.

The aim of this study is to test whether it is possible to increase the 2-month survival rate (null responder to steroids) or the 6-month survival rate (partial responder to steroids) using G-CSF treatment in patients with severe $\mathrm{AH}$ with high mortality despite corticosteroid treatment.

\section{Methods/design}

Study objectives

The aim of this study is to establish proof of concept whether patients with severe AH (MDF score $\geq 32$ ) would benefit from G-CSF rescue therapy. The primary aim is to evaluate whether G-CSF treatment prolongs 6-month overall survival (OS) of patients with a partial response (PR) to steroids and 2-month OS of patients with a null response (NR) to steroids. Partial responders $(0.16<$ Lille score $<0.56)$ or null responders (Lille score $\geq$ 0.56) are identified based on the Lille score after 1 week of corticosteroid treatment (40 mg daily prednisolone dose). The secondary aims are to identify the risk factors in relation to mortality and the predictive factors associated with responses to standard corticosteroid treatment or rescue G-CSF therapy. Pre-allocation and post-allocation risk factors related to mortality include age, sex, the presence of ascites, infection, gastrointestinal bleeding, daily dose of alcohol consumed, the presence and period of abstinence, white blood cell count, hemoglobin, hematocrit, high sensitivity C-reactive protein, blood urea nitrogen, creatinine, aspartate aminotransferase (AST), alanine aminotransferase (ALT), gamma-glutamyl transferase, albumin, and prothrombin time. The secondary aims include improvements in liver function (Child-Turcotte-Pugh (CTP) score, MELD score, and chronic liver failure sequential organ failure assessment (CLIF-SOFA)( score), changes in the 
percentage of CD34-positive cells in peripheral blood, and changes in hepatic histological findings.

\section{Sample size}

The 1-month OS rate in patients with severe $\mathrm{AH}$ undergoing steroid therapy has been reported as $80 \%$ and that in a control group as $66 \%$ [7]. Moreover, the 6-month OS rate of steroid responders has been reported as $85 \%$ and that of non-responders as $25 \%$ [13]. A previous, large-scale, randomized study compared co-administration of steroid and pentoxifylline with steroid monotherapy. The 6-month survival rate of the complete responders in the steroid monotherapy group was $90.5 \%$, whereas the 6-month survival rate of partial responders was $82 \%$ [20]. The 2-month survival rate was $66 \%$ in the G-CSF group and $26 \%$ in the control group in a previous study conducted in patients with hepatic failure, in whom $\mathrm{AH}$ was responsible for approximately $60 \%$ of cases of hepatic failure [14]. Additionally, the 2-month survival rate of null responders was 42\% [21]. Based on these data, the sample size was estimated using PASS 11.0 software (NCSS, Kaysville, UT, USA).

We hypothesized that there would be a significant difference in 6-month OS between steroid therapy and steroid plus G-CSF therapy in patients with a PR to steroids ( $82 \%$ vs. $90.5 \%)$. A two-sided sample size calculation with power of 0.8 , significance level of 0.05 , and estimated dropout rate of $10 \%$ revealed that at least 190 patients should be included in the PR study: 95 patients in the steroid plus placebo group and 95 in the steroid plus G-CSF group.

We hypothesized that there would be a significant difference in 2-month OS between placebo therapy and G-CSF therapy (42\% vs. $66 \%)$ for patients with a NR to steroids. A two-sided sample size calculation with power of 0.8 , significance level of 0.05 , and estimated dropout rate of $10 \%$ revealed that at least 78 patients should be included in the NR study: 39 patients in the placebo group and 39 in the G-CSF group.

\section{Trial design}

The current study is a prospective, double-blind, multicenter (15 centers), randomized, placebo-controlled trial with two parallel subgroups (PR and NR groups) (Fig. 1). We will evaluate the PR (Lille score 0.16-0.56) and NR (Lille score $\geq 0.56$ ) by calculating the Lille score after 1 week of steroid therapy in patients who meet the eligibility criteria. Partial responders are expected to have the OS benefits of steroid therapy, thereby, the subjects will be randomly assigned to either the steroid-placebo co-administration group or the steroid-G-CSF co-administration group (1:1). Study subjects in the PR group will be treated with prednisolone $40 \mathrm{mg} /$ day for 28 days followed by dose tapering.

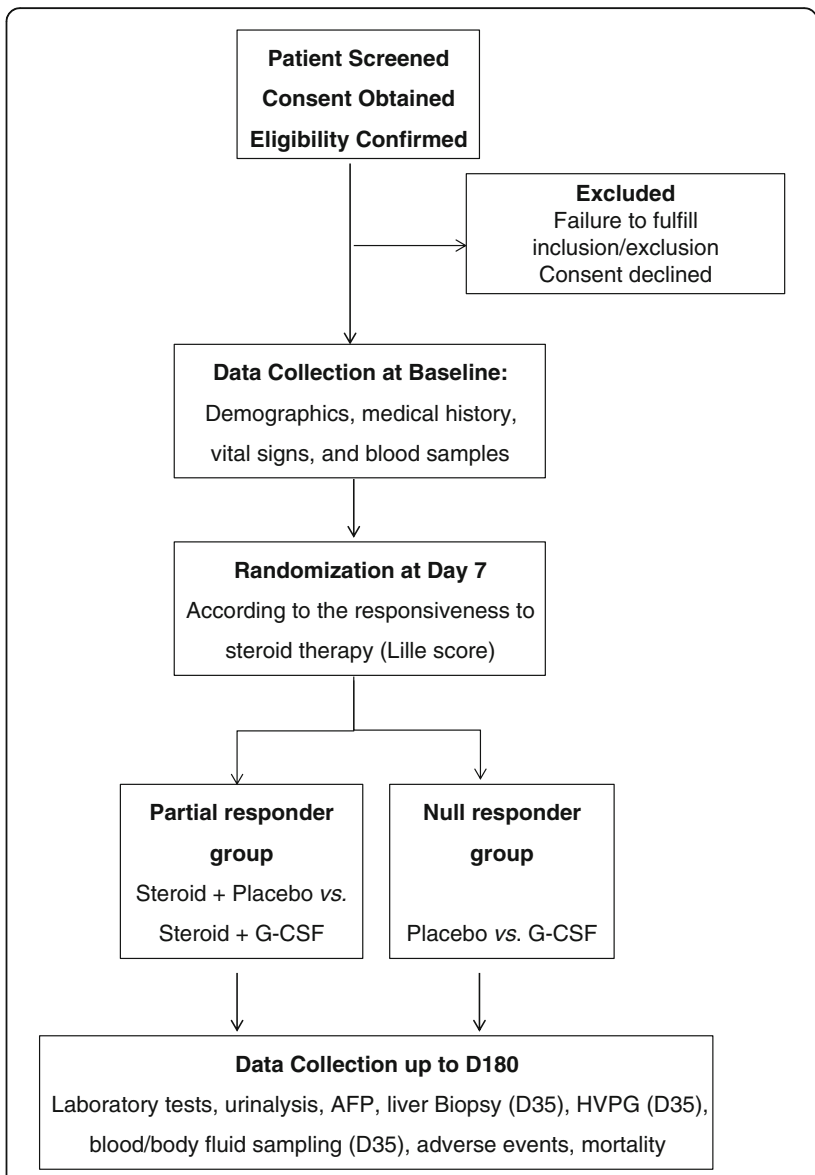

Fig. 1 Flowchart for study participants. Abbreviations: G-CSF, granulocyte colony stimulating factor; AFP, alpha-fetoprotein; HVPG, hepatic venous pressure gradient; $D$, day

Steroid therapy will be discontinued in the NR group because the clinical benefits from steroid therapy are not proven and there was concern over adverse effects of steroid therapy in the NR group. When liver transplantation is not feasible for NRs, they will be randomly assigned to either the placebo group or the G-CSF group (1:1).

A preliminary study including five subjects each from the steroid-G-CSF versus steroid-placebo groups in the PR group will be conducted to assess safety data according to the adverse event assessment criteria of the National Marrow Donor Program (NMDP) filgrastim-mobilized, peripheral-blood stem cell donor assessment for the solid validation on the safety of steroid-G-CSF co-administration [22]. The independent Data Safety Monitoring Board (DSMB) committee (a hepatologist, an oncologist, a gastroenterologist, and a biostatistician, who do not participate in this trial) will analyze the preliminary safety data according to the NMDP safety assessment criteria. The data from the preliminary study will be unblinded only to the DSMB committee, therefore will be included in the main study 
results. The trial follows the recommendations for interventional trials guidelines (SPIRIT; see Additional file 1).

\section{Interventions}

All study subjects are started on corticosteroid as standard therapy if they have no contraindications to corticosteroid treatment (prednisolone $40 \mathrm{mg}$ daily); partial responders will have their dose reduced after 28 days of administration, whereas null responders will stop the drug after 7 days administration. When oral dosing is not tolerable, 32 mg methylprednisolone will be administered via intravenous injection daily, which is a dosage with equivalent efficacy. G-CSF will be subcutaneously injected at a dosage of $5 \mu \mathrm{g} / \mathrm{kg}$ body weight daily for 5 days from the next day of randomization in the PR and NR groups. Thereafter, it will be administered at the same dosage every 3 days, for a total of 12 times (days of G-CSF administration, day 8-12, 15, 18, 21, 24, 27, 30, and 33) (Fig. 2).

\section{Study drug}

G-CSF (Leucostim ${ }^{\circledR}$ Injection, Dong-A ST Co., Ltd., Seoul, Korea, nomenclature of component filgrastim) is regarded as an investigational medicinal product for this study. The dose and volume of G-CSF will be determined on the first day according to the body weight of each subject (Table 1). G-CSF will be subcutaneously administered 12 times (daily for 5 days, followed by every 3 days for 7 times). Normal saline (JW Pharmaceutical, Seoul, Korea) will be used as a placebo at a maximal volume of $1.2 \mathrm{~mL}$.

\section{Inclusion criteria}

The inclusion criteria are as follows:

- Age $>20$ years and $<80$ years

- History of clinically significant amount of alcohol intake ( $\geq 50 \mathrm{~g}$ daily for men, and $\geq 40 \mathrm{~g}$ daily for women over the last 2 months)

\begin{tabular}{|c|c|c|c|c|}
\hline \multirow[b]{2}{*}{ TIME POINTS } & \multirow[b]{2}{*}{ Enrolment } & \multirow{2}{*}{\begin{tabular}{|c|} 
Post- \\
allocation \\
(D1,3,7,9, \\
$11,14,17$ \\
$20,23,26$ \\
$29,32,35)$ \\
\end{tabular}} & \multicolumn{2}{|l|}{ Follow-up } \\
\hline & & & $\mathrm{D} 60,90,120,150$ & D180 \\
\hline $\begin{array}{l}\text { ENROLMENT: } \\
\text { Eligibility screen } \\
\text { Informed Consent } \\
\text { Patient Demographics } \\
\text { Randomization } \\
\text { Treatment Group Allocation }\end{array}$ & : & & & \\
\hline $\begin{array}{l}\text { INTERVENTIONS: } \\
\text { (12 times during D8-33) } \\
\text { Partial response group: steroid+placebo } \\
\text { vs. steroid+G-CSF } \\
\text { Null response group: placebo vs. G-CSF }\end{array}$ & & $\begin{array}{l}0 \\
0\end{array}$ & $\begin{array}{l}0 \\
0\end{array}$ & $\begin{array}{l}0 \\
0\end{array}$ \\
\hline $\begin{array}{l}\text { ASSESMENTS: } \\
\text { Past medical history } \\
\text { Physical examination } \\
\text { AUDIT-K } \\
\text { Laboratory tests } \\
\text { Urinalysis } \\
\text { Clinical assessments } \\
\text { Culture study } \\
\text { Viral markers } \\
\text { Chest PA } \\
\text { Abdominal USG or CT or MRI } \\
\text { Endoscopy } \\
\text { Alpha-fetoprotein } \\
\text { Procalcitonin } \\
\text { Liver Biopsy } \\
\text { Hepatic venous pressure gradient } \\
\text { Blood/body fluid sampling } \\
\text { CTP score, MELD score, CLIF-SOFA } \\
\text { CD34-positive cells in peripheral blood } \\
\end{array}$ & 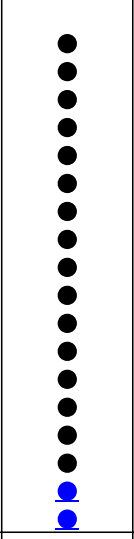 & \begin{tabular}{|}
$\mathbf{0}$ \\
(D35) \\
(D35) \\
(D7, 35) \\
(D7, 35) \\
(D7. 35) \\
\end{tabular} & - & • \\
\hline $\begin{array}{l}\text { OUTCOMES: } \\
\text { Partial response group: } 6 \text {-month survival } \\
\text { Null response group: } 2 \text {-month survival } \\
\text { Risk factors for mortality } \\
\text { Predictive factor for steroid response }\end{array}$ & & & 0 & 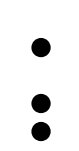 \\
\hline
\end{tabular}

Fig. 2 Study schedule of enrollment, intervention, and assessment. Abbreviations: G-CSF, granulocyte colony stimulating factor; AUDIT-K, Alchol Use Disorder Identification Test-Korea; USG, ultrasonography; CT, computed tomography; MRI, magnetic resonance imaging; CTP, Child-TurcottePugh, MELD, model for end-stage liver disease; CLIF-SOFA, chronic liver failure sequential organ failure assessment; $D$, day 
Table 1 Dose of G-CSF according to the body weight of each subject

\begin{tabular}{lll}
\hline Bodyweight $(\mathrm{kg})$ & Dose of G-CSF $(\mu \mathrm{g})$ & Volume $(\mathrm{mL})$ \\
\hline $40-44$ & 200 & 0.8 \\
$45-49$ & 225 & 0.9 \\
$50-54$ & 250 & 1.0 \\
$55-59$ & 275 & 1.1 \\
$\geq 60$ & 300 & 1.2 \\
\hline
\end{tabular}

G-CSF granulocyte colony stimulating factor

- MDF during the screening period $=[4.6 \times$ (patient's prothrombin time, sec - control prothrombin time, sec)] + serum bilirubin level $(\mathrm{mg} / \mathrm{dL}) \geq 32$

- New occurrence of jaundice within the last 3 months when a liver biopsy could not be performed, (total serum bilirubin level $>5 \mathrm{mg} / \mathrm{dL}$ ), or pathological transjugular liver tissue biopsy results consistent with alcoholic hepatitis (hepatocellular ballooning and polymorphonuclear leukocytic infiltration)

- After satisfying criteria $1-4$, Lille score $>0.16$ on day 7 after daily administration of $40 \mathrm{mg}$ prednisolone (or intravenous methylprednisolone at the equivalent efficacy dose in the case of oral intolerability)

\section{Exclusion criteria}

The exclusion criteria are as follows:

- Positive for the hepatitis B surface antigen, antihepatitis $\mathrm{C}$ antibody, or anti-human immunodeficiency virus antibody

- Malignant neoplasm including hepatocellular carcinoma

- $\mathrm{AST}>500 \mathrm{IU} / \mathrm{L}$ or ALT $>300 \mathrm{IU} / \mathrm{L}$

- Portal vein thrombosis, hemochromatosis, autoimmune hepatitis, Wilson's disease, or alpha-1antitrypsin deficiency

- Pregnant or breastfeeding women or those who refuse to use or cannot use contraceptives

- History of hypersensitivity to G-CSF injection

- Hypovolemic shock due to gastrointestinal bleeding at the time of hospitalization, or in need of a transfusion of more than three units of packed red blood cells, or MDF elevated from $<32$ points to $\geq$ 32 points due to gastrointestinal bleeding

- Sepsis or uncontrolled acute infection

- Hepatic encephalopathy grade 3-4

- Previous history treatment with corticosteroids or pentoxifylline within the past 3 months

- Myeloblasts observed in peripheral blood

- Severe comorbidities (type 1 hepatorenal syndrome or serum creatinine $>2.5 \mathrm{mg} / \mathrm{dL}$ at the time of screening, heart failure, lung disease, mental illness, or acute pancreatitis)

- Refusal to participate in the clinical trial

\section{Consent}

The investigator will explain the details of this clinical trial to potential study subjects, and provide sufficient time for them to consider whether they will participate in the study. Voluntary informed consent will be obtained from the study subjects in a written form. Consent will be documented as being signed and dated by the study subject on the informed consent form. Consent will be obtained in a written form from a parent or a legal guardian or a legally acceptable representative when a study subject is legally incompetent.

\section{Randomization and blinding procedures}

The study participants will be given a unique identification number, and they will be assigned to either the study-drug group or the control-placebo group. Randomization will be conducted using a web-based randomization program constructed by means of the blocked randomization method. The web-based randomization will be managed by the Medical Research Collaborating Center of Seoul National University Hospital (https://mrcc.snuh.org/) and stratification variables are individual centers and whether or not liver biopsy was performed.

The placebo and the study drug will be managed and supplied by the clinical pharmacy at each institution. A subject identification number will be given to each study subject in accordance with the randomization table, and the clinical pharmacy will provide the placebo or the study drug with the study subjects and the investigators blinded to allocation.

\section{Safety}

The principal investigator will observe adverse events (AEs) and all AEs spontaneously reported by the study subjects. The principal investigator will assess all AEs for seriousness, causality, severity, and for expectedness if the AE is related to the study drug. All AEs will be assessed by the principal investigator as possibly, probably, or definitely related to the study drug and all serious adverse events (SAEs) that occur during the study period will be followed until they are resolved or clearly determined to be due to a patient's stable or chronic conditions or intercurrent illness. Reporting will follow the filgrastim-mobilized, peripheral blood stem cell donor assessment of NMDP [23].

A SAE is defined as an $\mathrm{AE}$ that:

- Results in death

- Results in life-threatening events

- Results in persistent or significant disability or incapacity involving a congenital anomaly or birth defect 
- Represents any other important medical event that carries a real but not a hypothetical risk of one of the outcomes listed above

\section{Data collection and management}

An electronic clinical record form (eCRF; see Additional file 2) will be used to collect the data on each study subject. The study subjects will be identified on the eCRF through their unique trial identifier, allocated at the time of recruitment. Data will be coded at various stages during the study period. Data security and storage will follow the data management plan of the Data Safety Monitoring Board (DSMB). The DSMB plan will be developed by referring to the guidelines of the World Health Organization [24].

\section{Statistical analyses}

Intention-to-treat analysis will be used as the main assessment of efficacy. Per-protocol will be analyzed for the primary efficacy analysis (i.e., OS), as well as to ensure the stability of analytical outcomes. The OS is defined as the period from enrollment to death or the last follow-up date, and will be analyzed using Kaplan-Meier estimates. To test the difference in OS between the G-CSF and control groups, a log-rank test will be used, and the significance level will be $5 \%$ (two-sided test).

We will summarize the demographic and other baseline characteristics of the study participants. Continuous variables will be presented as mean \pm standard deviations or median (interquartile range). The two-sample $t$ test or Wilcoxon's rank sum test will be used to compare the groups according to the normal distribution. Categorical variables will be presented as frequency and the percentage. Pearson's chi-square test or Fisher's exact test will be used to compare the groups. Changes in the percentage of CD34-positive cells in peripheral blood and liver tissues at 1 month of treatment will be compared between the groups using the two-sample $t$-test or Wilcoxon's rank sum test. In the PR group, the changes in CTP score, MELD score, and CLIF-SOFA score at 1 week and $1,2,3,4,5$, and 6 months will be compared between the groups (steroid + placebo versus steroid + G-CSF) using the two-sample $t$ test or Wilcoxon's rank sum test. In the NR group, the changes in CTP score, MELD score, and CLIF-SOFA score at 1 week, 1 month, and 2 months will be compared between the groups (placebo vs. G-CSF) using two-sample $t$ test or Wilcoxon's rank sum test. In addition, repeated measures analysis of variance (ANOVA) will be performed to analyze the difference in repeated measures between the groups. OS of the subgroups according to the alcoholic hepatitis histologic score (AHHS) will be calculated by the Kaplan-Meier method and will be compared by log-rank test. Multivariate Cox regression analysis will be used to evaluate the independent variables $(P<0.05)$ in the whole study sample, the PR group, and the NR group. To determine the mortality prediction model, the cutoff value using area under the receiver operating characteristic curve (AUROC) will be calculated, and sensitivity and specificity will be calculated. The discrimination function of the model will be evaluated by the Kaplan-Meier method according to the estimated cut-off value. All analyses will be performed using SPSS Statistics software version 20.0 (IBM Corp., Armonk, NY, USA) and SAS version 9.2 software (SAS Institute, Cary, NC, USA).

\section{Discussion}

The purpose of this trial is to establish proof of concept of the efficacy of G-CSF in patients with histologically confirmed or clinically suspected, severe AH (MDF score $\geq 32$ ). Patients will be screened and enrolled into the PR (Lille score 0.16-0.56) and NR (Lille score $\geq 0.56$ ) groups by calculating the Lille score after 1 week of steroid therapy. Steroid therapy will be discontinued in null responders upon allocation to the NR group. They will be randomly assigned to either the placebo group or the G-CSF group (1:1). Partial responders are expected to have a survival benefit from steroid therapy, thereby the subjects will be randomly assigned to either the steroid-placebo co-administration group or the steroid-G-CSF co-administration group (1:1). Corticosteroids might reduce necroinflammation in the liver, but a high mortality rate is still noted in patients with severe who are $\mathrm{AH}$ receiving steroid therapy. Combining G-CSF with corticosteroid treatment might promote liver regeneration and improve neutrophil function, leading to the amelioration of clinical outcomes.

The Korean Association for the Study of the Liver clinical practice guidelines for the management of alcoholic liver disease (2013) recommend liver transplantation when there is no remarkable reduction in jaundice (Lille model score $\geq 0.56$ ) 1 week after steroid treatment is initiated [25]. However, in addition to a shortage of donor organs, it calls for pre-transplant 6-month abstinence from alcohol as a prerequisite for liver transplantation in most transplant centers; therefore, it is practically difficult to follow the recommendation of "considering a liver transplant" as viable.

The 6-month survival rate is higher in patients with severe $\mathrm{AH}$ who are receiving pentoxifylline than in those treated with placebo [26]. Pentoxifylline can be considered an alternative therapy for patients with severe $\mathrm{AH}$ and contraindication to corticosteroid treatment [27, 28]. A previous study reported the therapeutic efficiency of pentoxifylline in non-responders switching from corticosteroids [16]. Among the non-responders to corticosteroids, the investigators compared the 2-month survival rate in patients in whom corticosteroids were replaced with pentoxifylline at the early stage with that in those 
who had continuously taken corticosteroids; however, the 2-month survival rates were $35.5 \%$ and $31.0 \%$, respectively ( $P$ value not significant) [16]. Therefore, the switch to pentoxifylline is not clinically beneficial in non-responders to corticosteroids.

It is essential to develop a new treatment modality to overcome therapeutic refractoriness or resistance and improve survival of patients with severe $\mathrm{AH}$ who are refractory to steroid therapy. Some studies have reported that G-CSF stimulates proliferation of hepatocytes in patients with alcoholic $\mathrm{AH}$, cirrhosis, and liver failure, by mobilizing CD34-positive stem cells to the liver $[14,15]$. These studies enrolled a small number of subjects and the follow-up period was only 1 month. However, they suggest G-CSF may be beneficial in improving long-term survival in patients with severe $\mathrm{AH}$. Therefore, we hypothesize that G-CSF treatment might prolong the survival of patients with severe $\mathrm{AH}$ who are refractory to corticosteroid treatment. This is the first proof-of-concept trial designed to assess the efficacy of G-CSF according to the response to corticosteroids. This large-scale, multicenter, randomized controlled trial is also designed to find a special subgroup of patients in whom G-CSF effectively works to improve liver function and prolong survival.

\section{Trial status}

This trial is ongoing and actively recruiting. Recruitment started on 13 May 2015. Completion is anticipated on 31 December 2020.

\section{Additional files}

Additional file 1: SPIRIT checklist. (DOC $123 \mathrm{~kb}$ )

Additional file 2: Case Report Form. (PDF $911 \mathrm{~kb}$ )

\section{Abbreviations}

AFP: Alpha-fetoprotein; AH: Alcoholic hepatitis; AHHS: Acoholic hepatitis histologic sore; ALT: Alanine aminotransferase; ANOVA: Analysis of variance; AST: Aspartate aminotransferase; AUROC: Area under the receiver operating characteristic curve; CBC: Complete blood cell count; CLIF-SOFA: Chronic liver failure sequential organ failure assessment; CR: Complete response: DF: Discriminant function; EASL: European Association for the Study of the Liver; eCRF: Electronic clinical record form; G-CSF: Granulocyte colony stimulating factor; HVPG: Hepatic venous pressure gradient; ITT: Intention to treat; MDF: Maddrey's discriminant function; MELD: Model for end-stage liver disease; NMDP: National Marrow Donor Program; NR: Null response; OS: Overall survival; PR: Partial response

\section{Funding}

This study was supported by the 2014 Korean Association for the Study of the Liver Research Fund. The funder had no role in study design, data collection and analysis, decision to publish, or preparation of the manuscript.

\section{Availability of data and materials}

Details of data management procedures and eCRFs can be obtained from drwon1@snu.ac.kr. The datasets generated and/or analyzed during the current study are available upon reasonable request.

\section{Authors' contributions}

YC, YSP, WK, HJL, and DJK each contributed substantially to the study and participated in the design. All authors read and approved the final manuscript and agreed to be accountable for all aspects of the work.

\section{Ethics approval and consent to participate}

The trial has been approved by the Institutional Review Board (IRB) of Seoul National University Boramae Medical Center and by the IRBs of the 14 participating centers (Hallym University Medical Center-Chuncheon, Pusan National University Hospital, Pusan National University Yangsan Hospital, Daegu Catholic University Medical Center, Ulsan University Hospital, St. Vincent's Hospital, Korea University Ansan Hospital, Hanyang University Guri Hospital, Chonbuk National University Hospital, Yonsei University Wonju Severance Christian Hospital, GangNeung Asan Hospital, Uijeongbu St. Mary's Hospital, Wonkwang University Hospital, and Gyeongsang National University Hospital). Informed consent will be obtained from all participants in the study.

\section{Consent for publication}

Not applicable.

\section{Competing interests}

The authors declare that they have no competing interests.

\section{Publisher's Note}

Springer Nature remains neutral with regard to jurisdictional claims in published maps and institutional affiliations.

\section{Author details}

${ }^{1}$ Department of Internal Medicine, CHA Gangnam Medical Center, CHA University School of Medicine, Seoul, Republic of Korea. ${ }^{2}$ Division of Gastroenterology and Hepatology, Department of Internal Medicine, Seoul Metropolitan Government Seoul National University Boramae Medical Center, 20, Boramae-ro 5-gil, Dongjak-gu, Seoul 07061, Republic of Korea. ${ }^{3}$ Department of Internal Medicine, College of Medicine, Ewha Womans University, Seoul, Republic of Korea. ${ }^{4}$ Department of Internal Medicine, Yeungnam University College of Medicine, Yeungnam University Medical Center, 170, Hyeonchung-ro, Nam-gu, Daegu 42415, Republic of Korea. ${ }^{5}$ Department of Internal Medicine, Hallym University College of Medicine, Chuncheon Hallym University Medical Center, 153 Gyo-dong, Chuncheon-si, Gangwon-do 24253, Republic of Korea.

Received: 6 September 2018 Accepted: 30 November 2018

Published online: 22 December 2018

\section{References}

1. Rehm J, Samokhvalov AV, Shield KD. Global burden of alcoholic liver diseases. J Hepatol. 2013;59:160-8.

2. Jang JY, Kim DJ. Epidemiology of alcoholic liver disease in Korea. Clin Mol Hepatol. 2018;24:93-9.

3. Lucey MR, Mathurin P, Morgan TR. Alcoholic hepatitis. N Engl J Med. 2009: 360:2758-69.

4. Mathurin P, Duchatelle V, Ramond MJ, et al. Survival and prognostic factors in patients with severe alcoholic hepatitis treated with prednisolone. Gastroenterology. 1996;110:1847-53.

5. Kim WR, Brown RS Jr, Terrault NA, El-Serag H. Burden of liver disease in the United States: summary of a workshop. Hepatology. 2002;36:227-42.

6. Dominguez $M$, Rincon $D$, Abraldes JG, et al. A new scoring system for prognostic stratification of patients with alcoholic hepatitis. Am J Gastroenterol. 2008;103:2747-56.

7. Mathurin P, O'Grady J, Carithers RL, et al. Corticosteroids improve short-term survival in patients with severe alcoholic hepatitis: meta-analysis of individual patient data. Gut. 2011;60:255-60.

8. Liangpunsakul S. Clinical characteristics and mortality of hospitalized alcoholic hepatitis patients in the United States. J Clin Gastroenterol. 2011; 45:714-9.

9. Maddrey WC, Boitnott JK, Bedine MS, Weber FL Jr, Mezey E, White RI Jr. Corticosteroid therapy of alcoholic hepatitis. Gastroenterology. 1978;75:193-9.

10. Dunn W, Jamil LH, Brown LS, et al. MELD accurately predicts mortality in patients with alcoholic hepatitis. Hepatology. 2005;41:353-8. 
11. European Association for the Study of L. EASL clinical practical guidelines: management of alcoholic liver disease. J Hepatol. 2012;57:399-420.

12. O'Shea RS, Dasarathy S, McCullough AJ, Practice Guideline Committee of the American Association for the Study of Liver Disease and Practice Parameters Committee of the American College of Gastroenterology. Alcoholic liver disease. Hepatology. 2010;51:307-28.

13. Louvet A, Naveau S, Abdelnour M, et al. The Lille model: a new tool for therapeutic strategy in patients with severe alcoholic hepatitis treated with steroids. Hepatology. 2007;45:1348-54.

14. Garg V, Garg H, Khan A, et al. Granulocyte colony-stimulating factor mobilizes CD34(+) cells and improves survival of patients with acute-onchronic liver failure. Gastroenterology. 2012;142:505-12 e1.

15. Spahr L, Lambert JF, Rubbia-Brandt L, et al. Granulocyte-colony stimulating factor induces proliferation of hepatic progenitors in alcoholic steatohepatitis: a randomized trial. Hepatology. 2008;48:221-9.

16. Louvet A, Diaz E, Dharancy S, et al. Early switch to pentoxifylline in patients with severe alcoholic hepatitis is inefficient in non-responders to corticosteroids. J Hepatol. 2008;48:465-70.

17. Gaia S, Smedile A, Omede P, et al. Feasibility and safety of G-CSF administration to induce bone marrow-derived cells mobilization in patients with end stage liver disease. J Hepatol. 2006;45:13-9.

18. Singh V, Sharma AK, Narasimhan RL, Bhalla A, Sharma N, Sharma R. Granulocyte colony-stimulating factor in severe alcoholic hepatitis: a randomized pilot study. Am J Gastroenterol. 2014;109:1417-23.

19. Piscaglia AC, Shupe TD, Oh SH, Gasbarrini A, Petersen BE. Granulocytecolony stimulating factor promotes liver repair and induces oval cell migration and proliferation in rats. Gastroenterology. 2007;133:619-31.

20. Mathurin P, Louvet A, Duhamel A, et al. Prednisolone with vs without pentoxifylline and survival of patients with severe alcoholic hepatitis: a randomized clinical trial. JAMA. 2013;310:1033-41.

21. Mathurin P, Moreno C, Samuel D, et al. Early liver transplantation for severe alcoholic hepatitis. N Engl J Med. 2011;365:1790-800.

22. National Marrow Donor Program. Filgrastim mobilized PBSC days one, two, three and four donor assessment. 2014. https:/www.cibmtr.org/ DataManagement/TrainingReference/eLearning/Documents/710_sample.pdf.

23. Pulsipher MA, Chitphakdithai P, Miller JP, et al. Adverse events among 2408 unrelated donors of peripheral blood stem cells: results of a prospective trial from the National Marrow Donor Program. Blood. 2009;113:3604-11.

24. World Health Organization. Operational guidelines for the establishment and functioning of data \& safety monitoring boards. 2004. http://www.who. int/tdr/publications/documents/operational-guidelines.pdf.

25. Korean Association for the Study of the Liver. KASL clinical practice guidelines: management of alcoholic liver disease. Clin Mol Hepatol. 2013; 19:216-54.

26. Akriviadis E, Botla R, Briggs W, Han S, Reynolds T, Shakil O. Pentoxifylline improves short-term survival in severe acute alcoholic hepatitis: a doubleblind, placebo-controlled trial. Gastroenterology. 2000;119:1637-48.

27. Parker R, Armstrong MJ, Corbett C, Rowe IA, Houlihan DD. Systematic review: pentoxifylline for the treatment of severe alcoholic hepatitis. Aliment Pharmacol Ther. 2013:37:845-54.

28. Whitfield K, Rambaldi A, Wetterslev J, Gluud C. Pentoxifylline for alcoholic hepatitis. Cochrane Database Syst Rev. 2009;(4):CD007339.

\section{Ready to submit your research? Choose BMC and benefit from:}

- fast, convenient online submission

- thorough peer review by experienced researchers in your field

- rapid publication on acceptance

- support for research data, including large and complex data types

- gold Open Access which fosters wider collaboration and increased citations

- maximum visibility for your research: over $100 \mathrm{M}$ website views per year

At BMC, research is always in progress.

Learn more biomedcentral.com/submissions 